\title{
O IMPACTO DO ENOS NA VARIABILIDADE TEMPORAL DA PRECIPITAÇÃO PLUVIOMÉTRICA NA BACIA HIDROGRÁFICA DO RIO PIQUIRI-PR
}

\author{
Márcio Greyck Guimarães CORREA \\ Emerson GALVANI
}

\begin{abstract}
RESUMO
Esta pesquisa foi desenvolvida considerando os impactos causados pelo El Niño Oscilação Sul (ENOS) na variabilidade da precipitação. Apresenta uma proposta de uso de um modelo estatístico inferencial para a identificação da participação do fenômeno, por meio do Índice de Oscilação Sul (IOS), na variabilidade temporal da precipitação pluviométrica na bacia hidrográfica do rio Piquiri-PR. Para o desenvolvimento da pesquisa optou-se por analisar a ocorrência de ENOS por meio do IOS, com os dados disponibilizados pelo National Climate Centre da Austrália e dados mensais de precipitação pluviométrica do Instituto das Águas do Paraná para 41 postos pluviométricos com série histórica entre 1976 e 2010. Os resultados obtidos indicam que 31,4\% dos meses da série histórica analisada tiveram IOS negativo $\leq-7$, enquanto $21,1 \%$ obtiveram IOS positivo $\geq+7$ e $47,5 \%$ dos meses foram considerados neutros com relação ao IOS. Aplicou-se o modelo estatístico GAMLSS (Modelos Aditivos Generalizados para Posição, Escala e Forma) para identificar a influência do ENOS na precipitação média mensal. Observou-se uma defasagem de até um mês entre o registro dos desvios de IOS e a precipitação registrada na bacia hidrográfica do rio Piquiri. Identificou-se ainda uma diminuição na precipitação média mensal quando se aumenta em média uma unidade de IOS ao modelo inferencial. Essa diminuição mostrou-se variável entre as três áreas de drenagem consideradas no estudo.
\end{abstract}

Palavras-chave: IOS; Bacia hidrográfica; El Niño; La Niña; GAMLSS.

\section{ABSTRACT}

THE IMPACT OF EL NIÑO-SOUTHERN OSCILLATION ON THE RAINFALL TEMPORAL VARIABILITY IN THE PIQUIRI WATERSHED, PARANÁ STATE, BRAZIL. This research was developed considering the impacts caused by El Niño Southern Oscillation (ENOS) on the variability of precipitation. It presents a suggestion to use an inferential statistical model to identify the participation of such phenomenon on rainfall temporal variability in the Piquiri watershed (Paraná State, Brazil), based on the Southern Oscillation Index (SOI). The ENSO phenomenon was analyzed using Southern Oscillation Index data provided by the Australian National Climate Centre and monthly rainfall data from 41 rain gauge stations in the period from 1976 to 2010, provided by Instituto das Águas do Paraná. A total of $31.4 \%$ of the analyzed historical series showed a negative Southern Oscillation Index $(\leq-7)$, while $21.1 \%$ presented a positive Southern Oscillation Index ( $\geq+7)$, and $47.5 \%$ of the months were considered neutral regarding the Southern Oscillation Index. The Generalized Additive Models for Location, Scale and Shape (GAMLSS) were applied to identify the influence of ENSO on monthly rainfall average. A lag of up to one month was observed between Southern Oscillation Index deviations and rainfall in the Piquiri river watershed. Average monthly rainfall was found to decrease when each Southern Oscillation Index unit increases in the inferential model. This decrease varied between the three drainage areas considered in the study.

Keywords: Southern Oscillation Index; Watershed; El Niño; La Niña; Generalized Additive Models for Location, Scale and Shape. 


\section{INTRODUÇÃO}

O desenvolvimento das técnicas de observação do tempo meteorológico proporcionou um grande avanço para a climatologia como um todo. FERREIRA (2006) aponta que desde a década de 1960 os satélites meteorológicos estão em órbita terrestre, fornecendo uma vasta quantidade de dados observacionais. O aumento das estações meteorológicas e das observações atmosféricas auxiliou na identificação e compreensão da variabilidade climática, das teleconexões, e em decorrência, de fenômenos como ENOS (El Niño - Oscilação Sul).

SILVA \& SILVA (2012) explicam que a variabilidade climática, compreendida como os desvios em torno da média climática regional, é regulada em boa parte pela interação oceano-atmosfera e fazem uma análise das principais interações que influenciam o clima na América do Sul por meio das teleconexões, destacando ENOS.

A interação entre a temperatura da superfície do mar e diferenças barométricas em distintas regiões do globo é considerada a principal forçante da variabilidade climática global. AMBRIZZI (2003) destaca que essa dinâmica é responsável por produzir trens de ondas capazes de interferir na variabilidade climática de áreas distintas do globo terrestre.

Ao estudar a influência das teleconexões na precipitação do norte e nordeste brasileiro, REBOITA \& SANTOS (2014) utilizaram o fenômeno ENOS e anomalias da TSM no Atlântico Tropical para averiguar tal correlação e perceberam que o acoplamento desses fenômenos resultou em situações de ocorrência de precipitação diferenciada para as regiões estudadas, trazendo um novo entendimento sobre os efeitos do El Niño e La Niña sobre o norte e nordeste do Brasil.

O fenômeno ENOS pode causar variações na dinâmica climática e estar relacionado a períodos prolongados de excesso de chuva que causam alagamentos, deslizamentos e cheias dos rios; também é associado a longos períodos de seca que trazem sérios riscos ao abastecimento hídrico, causando deficiência hídrica e sérios danos socioambientais, como constatado por MOLION \& BERNARDO (2000), NERY (2005) e LIMBERGER \& SILVA (2016).

A previsão do ENOS tem avançado consideravelmente, assim como o entendimento de suas decorrências na paisagem e nos sistemas socioambientais. Sabe-se que o fenômeno ENOS influencia o clima do Brasil de forma diferenciada, como demonstrado por GRIMM et al. (2000) e COELHO et al. (2002), que identificaram uma correlação negativa entre El Niño e precipitação no norte e nordeste do Brasil, e incremento na precipitação pluvial no centro-sul do país.

Pesquisadores em diversas áreas têm se esforçado para identificar, quantificar e prever o fenômeno e sua influência nas diversas regiões do globo. Modelos estatísticos têm sido utilizados com grande frequência nestes estudos. Dentre os modelos utilizados, esta pesquisa propõe-se a aplicar um modelo aditivo generalizado para posição, escala e forma (GAMLSS).

Estudos na área de climatologia têm sido realizados a partir do uso do modelo GAMLSS. DEBELE et al. (2017) testaram o modelo na determinação de frequência de inundações. JEHANZAIB et al. (2020) quantificaram a influência das mudanças climáticas e atividades humanas nas secas hidrológicas em bacias hidrográficas, utilizando o mesmo modelo estatístico. DANTAS et al. (2020) verificaram o uso do modelo na previsão das chuvas sobre o estado da Paraíba e relataram a importância da temperatura da superfície do mar (TSM) do Atlântico e Pacífico na distribuição das chuvas na região.

Considerando os impactos causados pela variabilidade da precipitação em decorrência do ENOS, este trabalho apresenta uma proposta de uso de um modelo estatístico (GAMLSS) para a identificação da participação do fenômeno, por meio do Índice de Oscilação Sul (IOS), na variabilidade temporal da precipitação pluviométrica na bacia hidrográfica do rio Piquiri, Estado do Paraná, Brasil.

\subsection{Localização da área de estudo}

A bacia hidrográfica do rio Piquiri está localizada numa região de transição climática entre o clima tropical e subtropical, como apresentado por CORREA (2013) (Figura 1).

A bacia hidrográfica é uma importante unidade de análise da paisagem, onde a água é o elemento responsável por dinamizar os processos naturais; é na bacia hidrográfica que o ciclo hidrológico pode ser melhor compreendido a partir dos processos de precipitação e escoamento da água. De acordo com RODRIGUES \& ADAMI (2005, p. 147), a bacia hidrográfica pode ser considerada: 

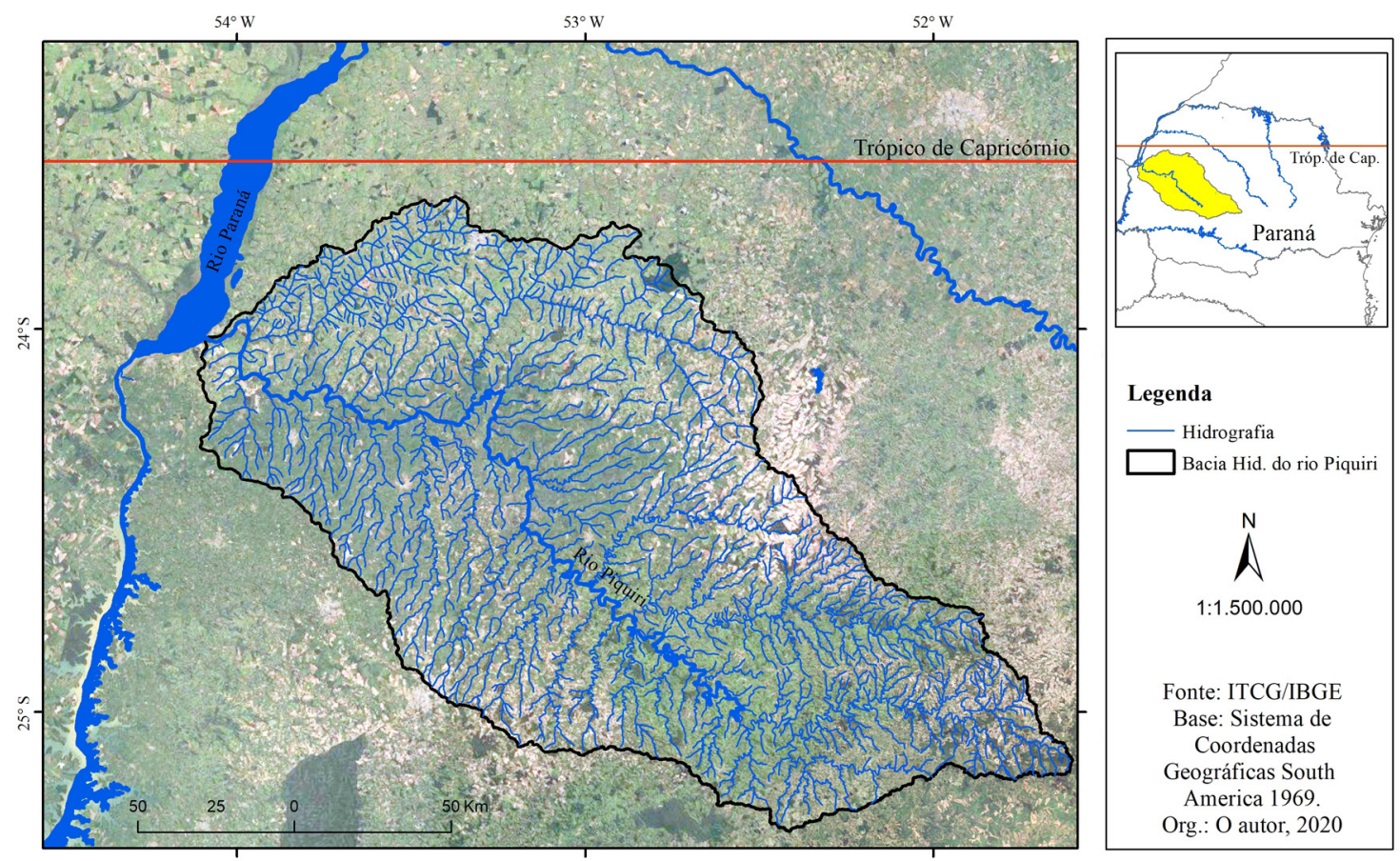

FIGURA 1 - Mapa de localização da bacia hidrográfica do rio Piquiri-PR.

\section{[...] um sistema que compreende um} volume de materiais, predominantemente sólidos e líquidos, próximos à superfície terrestre, delimitado interno e externamente por todos os processos que, a partir do fornecimento de água pela atmosfera, interferem no fluxo de matéria e de energia de um rio ou de uma rede de canais fluviais. Inclui, portanto, todos os espaços de circulação, armazenamento, e de saídas de água e do material por ela transportado, que mantêm relações com esses canais.

A bacia hidrográfica do rio Piquiri está localizada numa importante região agrícola do estado do Paraná, abrangendo 69 municípios do centro-oeste do estado, que em sua maioria são importantes produtores de grãos. Segundo o censo demográfico do IBGE (2012) a região tem uma população de aproximadamente 1,5 milhão de habitantes e, de acordo com o censo agropecuário IBGE (2006), a produção agrícola total é de mais de 2,2 bilhões de reais.

De acordo com a SEMA (2010), a demanda hídrica da Bacia do Piquiri é de aproximadamente $3000 \mathrm{~L} \mathrm{~s}^{-1}$, dos quais $52 \%$ provém de mananciais superficiais e $48 \%$ de mananciais subterrâneos. Os setores que mais usam a água da bacia são: abastecimento público (36\%), pecu- ária (31\%), indústrias (23\%), agricultura (11\%) e a mineração $(<1 \%)$.

A disponibilidade dos recursos hídricos é essencial para a economia dos municípios da região. A agricultura possui uma dependência indireta da água, uma vez que a estrutura fundiária e a organização do espaço agrário da bacia hidrográfica do Piquiri (predominantemente de pequenas propriedades) não faz uso em larga escala da irrigação, por isso a agricultura consome apenas $11 \%$ de água, conforme apresentado anteriormente. Portanto, a variabilidade das chuvas é um fator determinante para a manutenção da produção agrícola dessas áreas.

Conhecer a influência do fenômeno ENOS na distribuição da precipitação pluviométrica e na dinâmica hídrica do rio Piquiri tem um importante peso socioeconômico para estes municípios, uma vez que traz as possibilidades de adaptação e planejamento dos órgãos competentes.

\section{MATERIAIS E MÉTODOS}

\subsection{Escolha dos dados de IOS}

Para o desenvolvimento da pesquisa optou-se por analisar a ocorrência de ENOS por meio do Índice de Oscilação Sul (IOS), com os 
dados disponibilizados pelo National Climate Centre da Austrália. O critério adotado para a classificação dos meses baseaou-se na metodologia Troup SOI do Serviço de Meteorologia da Austrália (BOM 2020).

De acordo com essa metodologia de análise, consideram-se os meses de ocorrência de IOS negativo (El Niño) $<-7$ e IOS positivo (La Niña) $>+7$. Os valores de IOS apresentados pelo órgão responsável foram multiplicados por 10 (por convenção) e tem valores máximos variando entre $-35 \mathrm{e}+35$. O cálculo para a obtenção dos valores normalizados de IOS é apresentado na equação 1 .

$$
S O I=10 \cdot \frac{(\text { Pdiff }- \text { Pdiffav })}{S D(\text { Pdiff })}(\text { Eq. } 1)
$$

SOI: Índice de Oscilação Sul (IOS)

Pdiff: Diferença entre a pressão atmosférica medida no Taiti e Darwin para o mês.

Pdiffav: Média de Pdiff para o mês em questão.

$S D$ (Pdiff): Desvio padrão de Pdiff para o mês em questão.
2.2 Escolha dos dados de precipitação pluviométrica e determinação das áreas de drenagem

Para a análise da precipitação utilizou-se de dados secundários de precipitação pluviométrica do Instituto das Águas do Paraná, do qual se escolheu uma quantidade representativa de 41 postos pluviométricos na bacia hidrográfica do rio Piquiri, com série histórica de 35 anos entre 1976 e 2010.

No desenvolvimento da pesquisa determinou-se três grandes áreas de drenagem para a bacia hidrográfica do rio Piquiri. Cada área de drenagem foi definida de acordo com o sistema de escoamento e rede de drenagem, tendo como ponto de referência um posto fluviométrico da Agência Nacional de Águas (ANA). Assim a Área de drenagem 1 (A1), que abrange uma área de $1.690 \mathrm{~km}^{2}$, integra o posto fluviométrico de Guampará e mais 3 postos pluviométricos; a Área 2 (A2), com $4.160 \mathrm{~km}^{2}$, conta com a área de drenagem do posto Pto. Guarani e mais 6 postos pluviométricos; a Área 3 (A3), com 20.900 km², abrange a área de drenagem do posto de Balsa Santa Maria e 36 postos pluviométricos, sendo a área mais representativa da bacia, como mostra a figura 2. Desta forma, procurou-se correlacio-

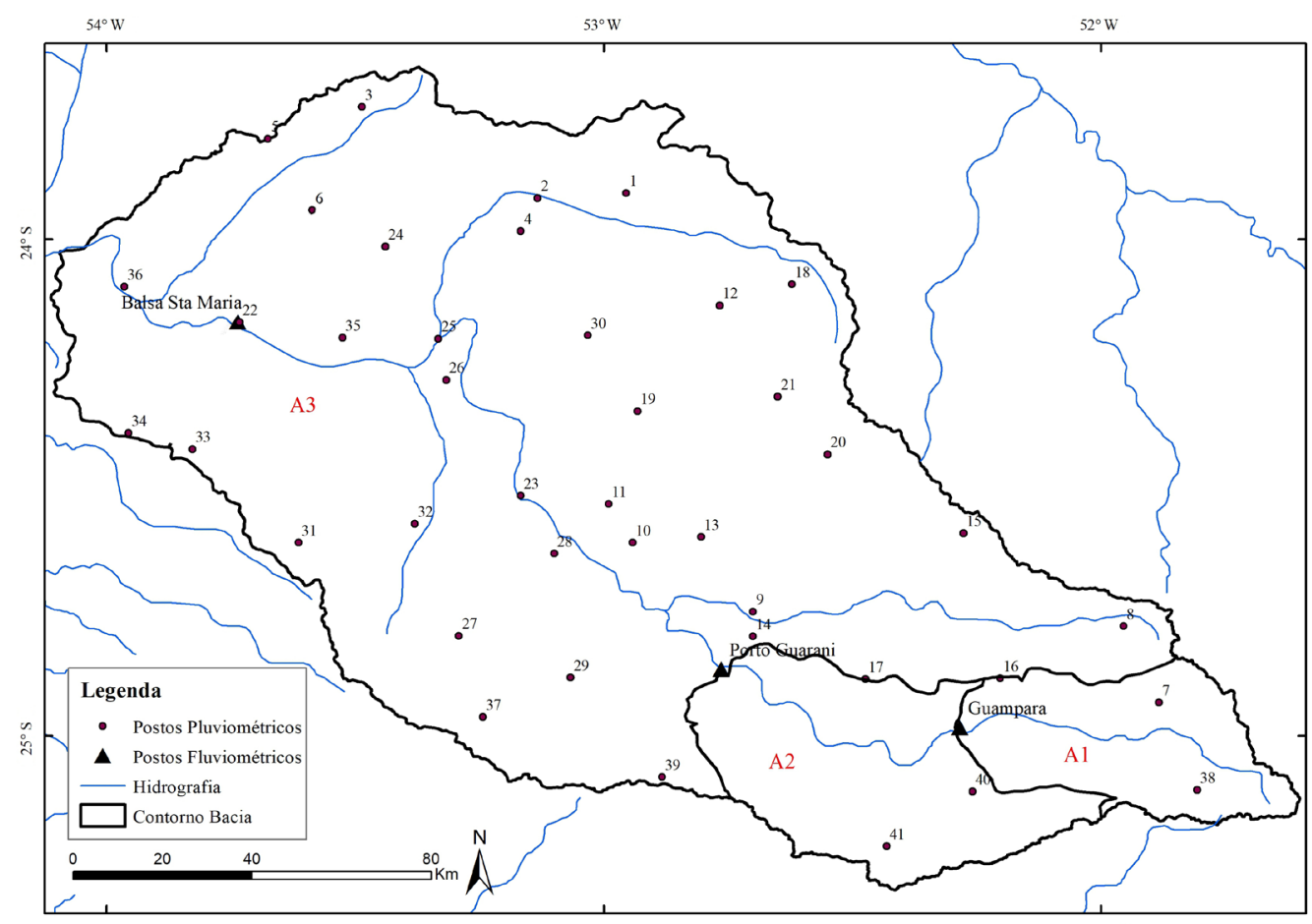

FIGURA 2 - Área de drenagem de cada posto fluviométrico na bacia hidrográfica do rio Piquiri, A1 - Guampará, A2 - Porto Guarani e A3 Balsa Santa Maria. 
nar o IOS e a precipitação pluviométrica de cada mês para cada posto pluviométrico em três setores distintos da bacia hidrográfica, alto, médio e baixo curso do rio Piquiri.

2.3 Análise e modelo estatístico empregado na pesquisa

Segundo LANNA (2001) a precipitação é uma variável hidrológica aleatória, o que dificulta conhecer a evolução destes fenômenos ao longo do tempo e do espaço; estatisticamente a correlação entre a precipitação e o IOS foi feita por meio de correlação linear de Pearson. Esse tipo de análise estatística permite observar a intensidade da correlação entre as variáveis estudadas, como indicado na equação 2 .

$$
r=\frac{\sum\left(x_{i}-\bar{x}\right)\left(y_{i}-\bar{y}\right)}{\sqrt{\left(\sum\left(x_{i}-\bar{x}\right)^{2}\right)\left(\sum\left(y_{i}-\bar{y}\right)^{2}\right)}}
$$

Em que: $x_{i}$ e $y_{i}$ indicam as duas variáveis observadas em cada passo de tempo $i, \mathrm{e}, \bar{x} \mathrm{e}$ $\bar{y}$ a média aritmética de cada série temporal; $\sqrt{\left(\sum\left(\mathrm{x}_{\mathrm{i}}-\overline{\mathrm{x}}\right)^{2}\right)\left(\sum\left(\mathrm{y}_{\mathrm{i}}-\bar{y}\right)^{2}\right)}$ corresponde ao desvio padrão de $x$ e $y$.

Sabe-se que existe um atraso entre a ocorrência de fenômenos sobre os oceanos e suas decorrências na precipitação pluviométrica no continente, como demonstrado por SCHNEIDER \& GIES (2004). Esse lag foi considerado na presente pesquisa e, para isso, realizou-se o teste de correlação linear para averiguar qual a melhor correlação entre as variáveis consideradas, apresentadas em um gráfico de autocorrelação cruzada.

Para caracterizar o efeito do IOS na bacia hidrográfica do rio Piquiri, utilizou-se modelos de regressão para modelar a média e, em algumas situações, a variância da variável precipitação. Sendo assim, buscou-se um procedimento que priorizasse identificar o impacto do IOS na precipitação média, um modelo de diagnóstico e não de previsão.

Optou-se por utilizar o modelo GAMLSS (Generalized Additive Model for Location, Scale and Shape - Modelos Aditivos Generalizados para Posição, Escala e Forma). De acordo com STASINOPOULOS \& RIGBY (2007), o GAMLSS é um tipo de modelo de regressão semi-paramétrico, que surgiu como uma abordagem para superar algumas limitações dos Modelos Lineares Generalizados (MLG) e dos Modelos Aditivos Generalizados (MAG).
O funcionamento deste modelo é descrito por MILHORANÇA (2014, p. 12):

"Com o GAMLSS podemos modelar diretamente todos os parâmetros da distribuição condicional de y, e não somente a média (localização). Para a maioria das distribuições os parâmetros são: média, variância, assimetria e curtose. Isso permite que possamos explicar a heteroscedasticidade por meio de variáveis explicativas, porém não permite ter parâmetros para a autocorrelação."

Com o modelo proposto esperou-se encontrar alguma resposta por meio da variável (precipitação) a influência do IOS na bacia hidrográfica do rio Piquiri. Os resíduos do modelo, ou seja, "a diferença entre o valor observado, e o valor esperado" (MILHORANÇA 2014, p. 13) foram transformados estatisticamente para terem distribuição normal padrão.

O modelo aplicado para a precipitação pode ser observado na equação 3 :

$$
\begin{gathered}
P_{t} \sim G G\left(\mu_{t}, \sigma_{t}, v\right) \text { independentes } \\
\log \left(\mu_{t}\right)=\log \left(E\left[P_{t}\right]\right)=\mu+\alpha_{M S_{t}}+\beta \operatorname{IOS}_{t-1}, \\
\log \left(\sigma_{t}\right)=\tau+\gamma_{M s_{t}}+\beta_{2} \operatorname{IOS}_{t-1} \\
\operatorname{Var}\left[P_{t}\right]=\left(\mu_{t} \times \sigma_{t}\right)^{2} .
\end{gathered}
$$

Pt: Precipitação da t-ésima observação.

$\mu$ : Parâmetro que representa a Precipitação média associada dos meses de janeiro.

$\alpha_{\text {Mest }_{t}}$ : Parâmetro que representa a diferença entre a Precipitação média dos meses correspondentes ao mesmo mês da t-ésima observação e a Precipitação média dos meses de janeiro.

$\beta$ : Parâmetro que representa a variação na média da Precipitação proporcionada pelo aumento de uma unidade de IOS.

O modelo GAMLSS utilizou dados de precipitação das três áreas de drenagem $\mathrm{A} 1, \mathrm{~A} 2 \mathrm{e}$ A3, produzindo os gráficos com os resíduos resultantes da modelagem.

Nos gráficos de resíduos espera-se que a dispersão dos pontos fique alinhada à reta, no intervalo de confiança, e que nos demais gráficos de dispersão os pontos estejam dispersos com amplitude constante em torno de zero, pois isso significa que o modelo se ajusta aos dados, de acordo com PAIVA et al. (2008). 
As tabelas com o resultado da estatística do modelo GAMLSS apresentam a estimativa, erro padrão, estatística t, valor-p e efeito para cada parâmetro utilizado na modelagem. Para a interpretação do modelo são essenciais os parâmetros: estatística t, valor-p e efeito.

\section{ANÁLISE E DISCUSSÃO DOS RESULTADOS}

3.1 Determinação da defasagem entre o IOS e sua influência na precipitação da bacia hidrográfica do rio Piquiri e análise residual do modelo GAMLSS

Considerando os dados de precipitação e IOS, de 420 meses da série histórica estudada (1976-2010), identificou-se 132 meses com IOS $\leq-7$ ( $31,4 \%$ do total de meses), enquanto 89 meses obtiveram IOS positivo $\leq+7(21,1 \%$ do total de meses), isso significa que $47,5 \%$ dos meses foram considerados neutros com relação ao IOS.

A influência do IOS no clima não ocorre instantaneamente, existe uma defasagem de tempo para que os efeitos das teleconexões sejam perceptíveis no clima de uma região. SILVA (2012) verificou a correlação entre a vazão dos rios do centro-norte do Pantanal e a TSM do Pacífico e concluiu que a defasagem mais confiável foi de até 4 meses; baseando-se nesse pressuposto, optou-se por realizar as correlações entre IOS e precipitação considerando as defasagens.

As correlações com defasagem apresentadas nas figuras 3, 4 e 5 (A1, A2 e A3, respectivamente) mostram que os valores da correlação são baixos em todas as defasagens e a maior correlação encontrada foi com defasagem de um mês (aproximadamente -0,1) para a precipitação. Com defasagens maiores que 10 meses também se observou correlações próximas de 0,1 , porém com pouca significância.

Ainda nas figuras 3, 4 e 5, pode-se observar o mesmo padrão de correlação e dispersão para as três áreas de drenagem (A1, A2 e A3). Com um mês de defasagem, os gráficos de dispersão mostram que a reta ajustada aos pontos é mais íngreme do que nos gráficos sem defasagem e os pontos apresentam-se mais agrupados em torno da reta. A defasagem de um mês entre IOS e precipitação é a mais confiável.

Importante destacar que a partir do décimo mês de defasagem as correlações mudam de sinal, porém poucos meses apresentaram resulta-
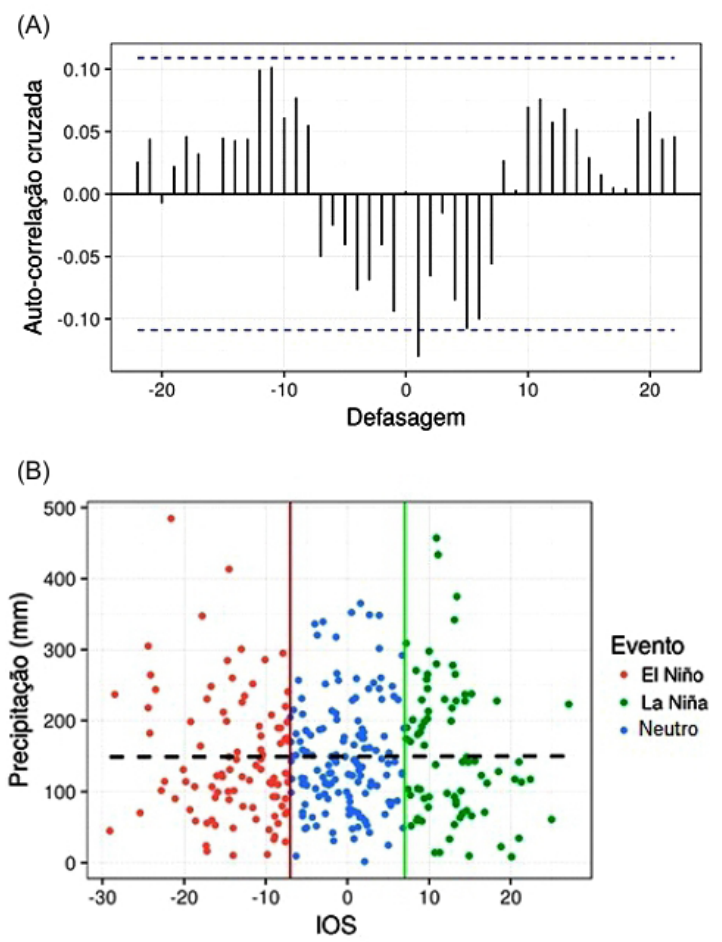

(C)

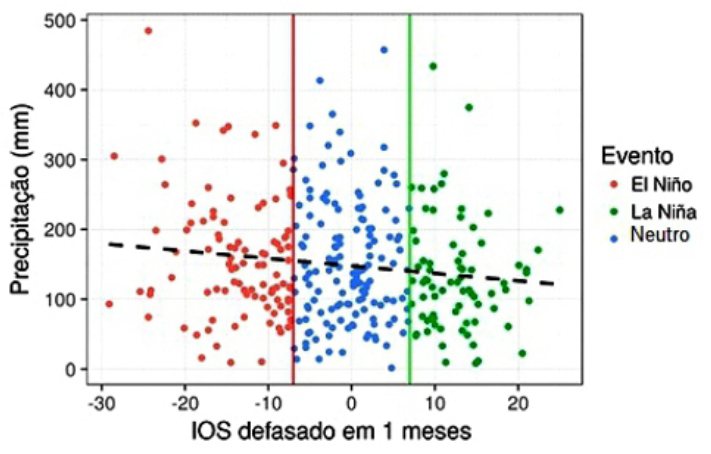

FIGURA 3 - Gráficos de dispersão e autocorrelação com defasagem entre precipitação e IOS Para Área 1- Guampará na bacia hidrográfica do rio Piquiri-PR. (A) Autocorrelação com defasagem entre Precipitação e IOS, (B) Gráfico de dispersão entre Precipitação e IOS mensal, (C) Gráfico de dispersão entre Precipitação e IOS defasagem de 1 mês.

dos confiáveis. Neste caso há que se considerar a persistência das correlações negativas de até sete meses, e maior confiança até o terceiro mês, o que corrobora ainda mais para a confiabilidade dos testes.

As correlações negativas podem ser consideradas mais confiáveis porque a maioria dos meses analisados apresentaram desvios de IOS negativos, indicando aquecimento das águas superficiais 
(A)

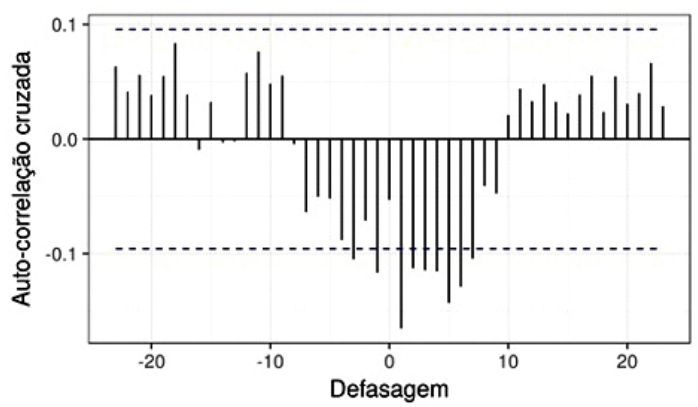

(B)

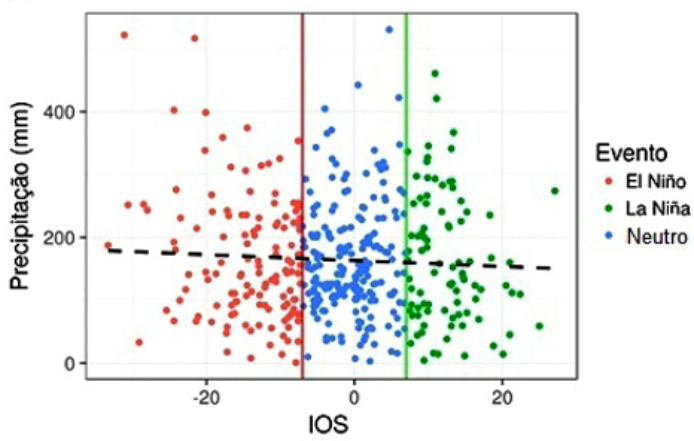

(C)

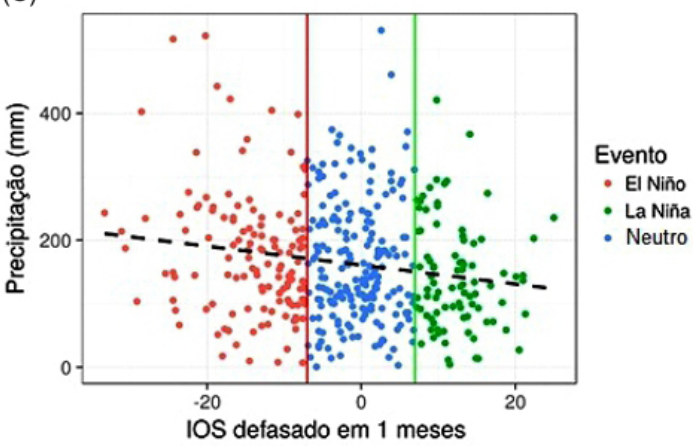

FIGURA 4 - Gráficos de dispersão e autocorrelação com defasagem entre precipitação e IOS Para Área 2Porto Guarani na bacia hidrográfica do rio Piquiri-PR. (A) Autocorrelação com defasagem entre Precipitação e IOS, (B) Gráfico de dispersão entre Precipitação e IOS mensal, (C) Gráfico de dispersão entre Precipitação e IOS defasagem de 1 mês.

do Pacífico e configuração da fase quente do fenômeno (El Niño), o que resultaria em maiores desvios positivos de chuva, grandezas inversamente proporcionais. Isso fica ainda mais evidente nos gráficos de dispersão para as três áreas consideradas com a reta inclinada em direção aos meses com IOS positivo (La Niña).

A aplicação do modelo GAMLSS gerou os gráficos de análise de resíduo para cada área
(A)

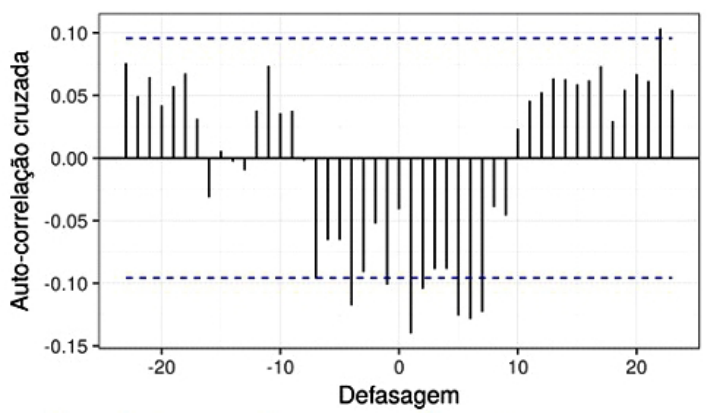

(B)

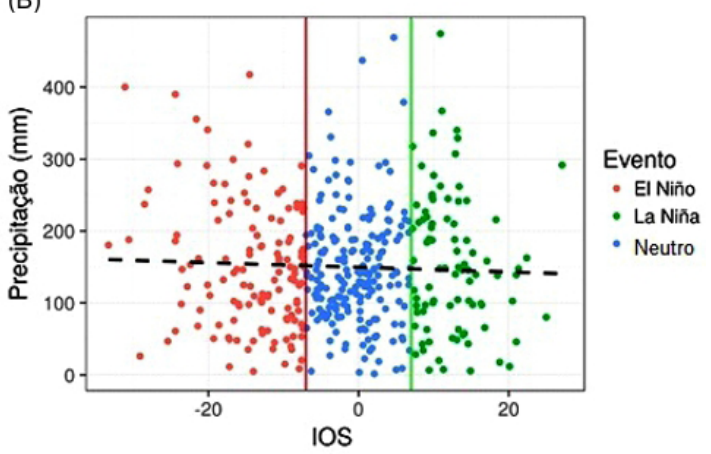

(C)

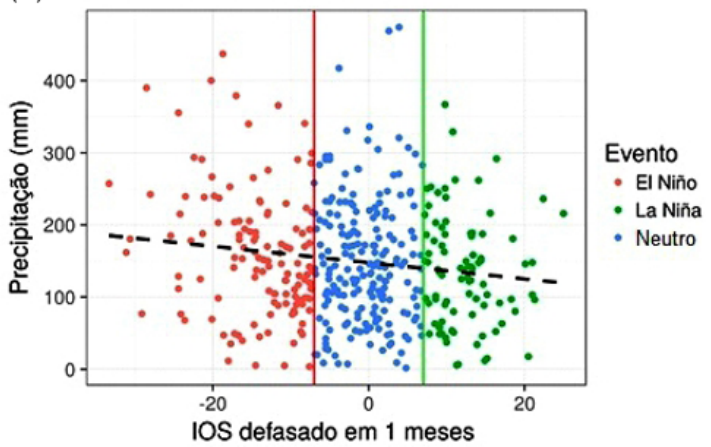

FIGURA 5 - Gráficos de dispersão e autocorrelação com defasagem entre precipitação e IOS Para Área 3- Balsa Santa Maria na bacia hidrográfica do rio Piquiri-PR. (A) Autocorrelação com defasagem entre Precipitação e IOS, (B) Gráfico de dispersão entre Precipitação e IOS mensal, (C) Gráfico de dispersão entre Precipitação e IOS defasagem de 1 mês.

de drenagem, conforme ilustrado pelas figuras 6,7 e 8 . Nota-se que as medidas de diagnóstico obtidas para esse modelo são relativamente satisfatórias, os resíduos padronizados estão normalmente distribuídos (dentro da área cinza) e a dispersão dos dados se alinha à reta, demonstrando homocedasticidade para A1 Guampará, A2 - Porto Guarani e A3 - Balsa Santa Maria. 

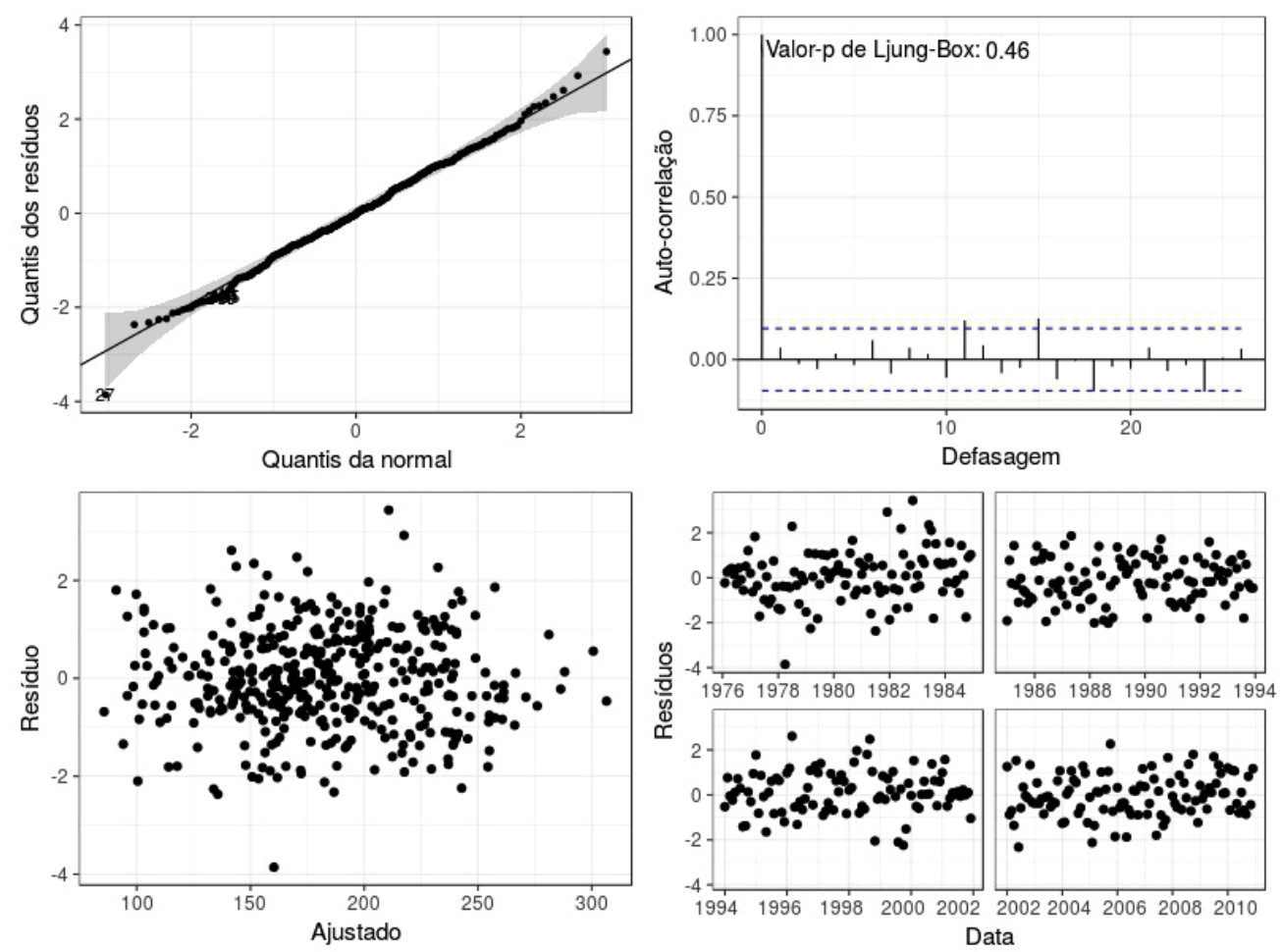

FIGURA 6 - Análise de resíduos do modelo GAMLSS duplo com resposta gama generalizada ajustado para a variável Precipitação em A1 - Guampará.
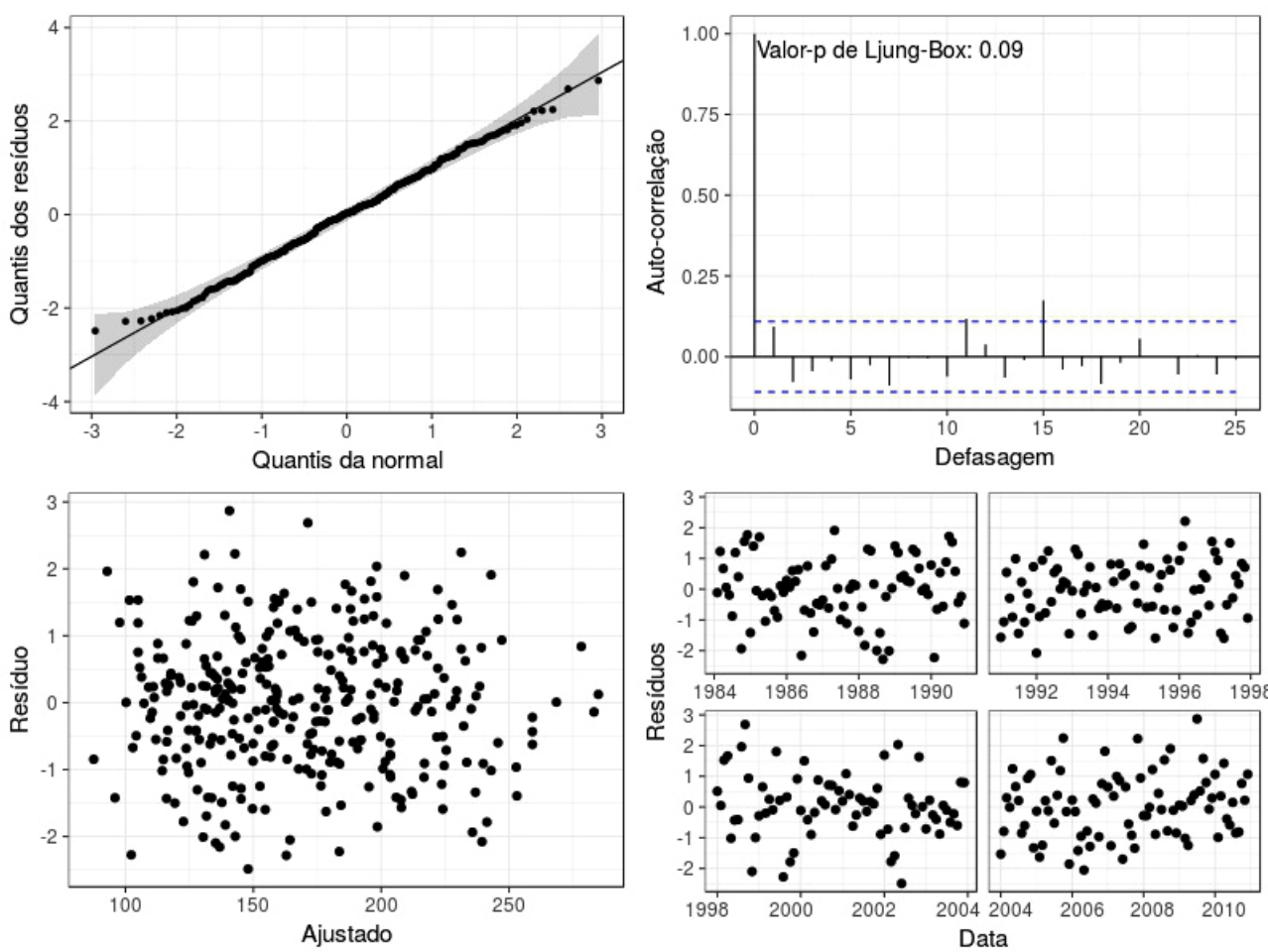

FIGURA 7 - Análise de resíduos do modelo GAMLSS duplo com resposta gama generalizada ajustado para a variável Precipitação em A2 - Porto Guarani. 

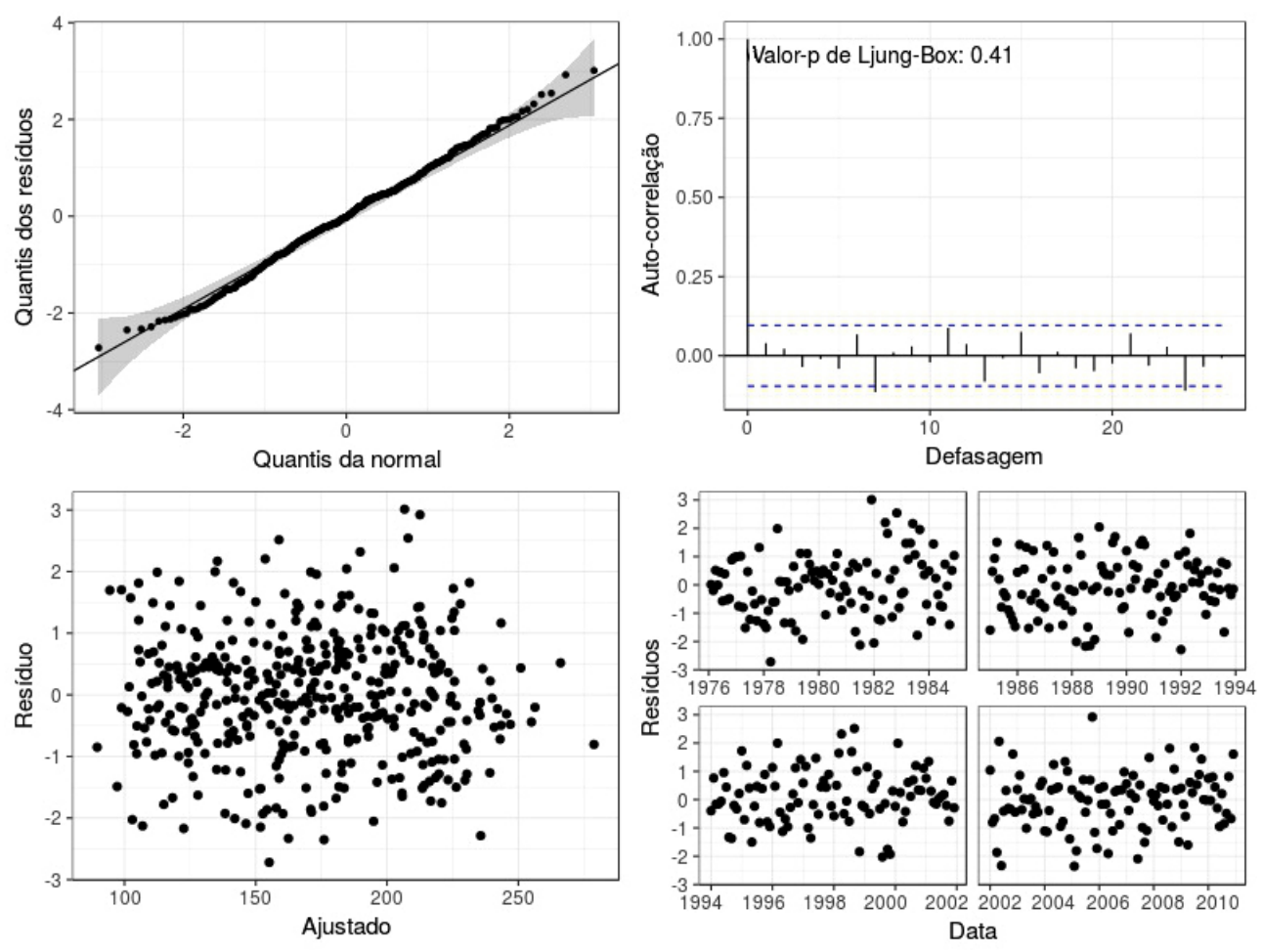

FIGURA 8 - Análise de resíduos do modelo GAMLSS duplo com resposta gama generalizada ajustado para a variável Precipitação em A3 - Balsa Santa Maria.

3.2 Resultados do modelo GAMLSS na identificação do impacto do ENOS na bacia hidrográfica do rio Piquiri

As tabelas 1, 2 e 3 mostram os parâmetros ajustados para a modelagem da média da precipitação no modelo GAMLSS com resposta Gama Generalizada para as três áreas de drenagem consideradas na pesquisa. Verificase uma redução estatisticamente significativa de $-0,99 \%$ para $\mathrm{A} 1$, de $-1,01 \%$ para $\mathrm{A} 2$ e de $-0,89 \%$ para A3 na média da precipitação para o aumento de uma unidade de IOS, ou seja, conforme aumenta uma unidade no IOS (tende a ser positivo - indicador da fase fria), a precipitação média diminui.

GRIMM et al. (2000) analisaram a variabilidade da precipitação na América do Sul associada ao El Niño e La Niña e apontaram que a região sul do Brasil apresenta maiores sinais de relação com o fenômeno, porém essa linearidade dos dados observados apresenta elevada variabilidade espacial da influência dos eventos de ENOS sobre a região.

Importante ressaltar que as áreas de drenagem A1 e A2 apresentaram maior efeito na redu- ção de precipitação, conforme aumenta uma unidade no IOS. Essas áreas se encontram mais ao sul da bacia hidrográfica do rio Piquiri, numa região que de acordo com a classificação climática de KÖPPEN (1948) aprimorada pelo IAPAR (1978) apresenta o tipo climático transitório entre $\mathrm{Cfb}$ (com temperatura média no mês mais frio abaixo de $18^{\circ} \mathrm{C}$, mesotérmico e com verões frescos sem estação seca definida) nas áreas mais elevadas e Cfa (clima subtropical, mesotérmico com verões quentes e geadas pouco frequentes). A área de drenagem A3 abrange uma área maior, inclusive o centro-norte da bacia hidrográfica, climaticamente mais quente e sazonalidade hídrica mais definida, características do clima tropical, classificada como tipo climático do tipo Cwa (caracteristicamente com chuvas de verão e estações bem definidas - verão-inverno - e temperaturas médias no mês mais quente superior a $22^{\circ} \mathrm{C}$ ).

Verifica-se que os meses de março, abril, junho, julho e agosto tem média de precipitação entre $30 \% \mathrm{e} 40 \%$ menor com relação à média da precipitação observada nos meses de janeiro. Para os demais meses, a variação identificada não foi significativa ao nível de $5 \%$. De acordo com CORREA \& GALVANI (2019) os períodos/meses citados 
TABELA 1 - Parâmetros ajustados para a modelagem da média da precipitação no modelo GAMLSS com resposta Gama Generalizada para A1 - Guampará.

\begin{tabular}{crrrrrr}
\hline Parâmetro Estimativa & $\begin{array}{c}\text { Erro } \\
\text { padrão }\end{array}$ & Estatística $t$ & Valor-p & Efeito \\
\hline$\mu$ & 5,3133 & 0,0722 & 73,5664 & $<0,0001$ & - \\
$\beta$ & $-0,0099$ & 0,0024 & $-4,0611$ & $<0,0001$ & $-00.99 \%$ \\
$\alpha \mathrm{Fev}$ & $-0,1113$ & 0,1165 & $-0,9553$ & 0,3402 & $-10.53 \%$ \\
$\alpha \mathrm{Mar}$ & $-0,4278$ & 0,0986 & $-4,3393$ & $<0,0001$ & $-34.81 \%$ \\
$\alpha$ Abr & $-0,3561$ & 0,1074 & $-3,3157$ & 0,0010 & $-29.96 \%$ \\
$\alpha \mathrm{Mai}$ & $-0,0613$ & 0,1335 & $-0,4590$ & 0,6465 & $-05.95 \%$ \\
$\alpha$ Jun & $-0,4597$ & 0,1231 & $-3,7338$ & $<0,0001$ & $-36.85 \%$ \\
$\alpha$ Jul & $-0,3895$ & 0,1285 & $-3,0315$ & 0,0026 & $-32.26 \%$ \\
$\alpha$ Ago & $-0,6383$ & 0,1509 & $-4,2311$ & $<0,0001$ & $-47.18 \%$ \\
$\alpha$ Set & $-0,0729$ & 0,1321 & $-0,5522$ & 0,5812 & $-07.03 \%$ \\
$\alpha$ Out & 0,1690 & 0,1130 & 1,4959 & 0,1357 & $18.41 \%$ \\
$\alpha$ Nov & $-0,2984$ & 0,1052 & $-2,8377$ & 0,0048 & $-25.80 \%$ \\
$\alpha$ Dez & $-0,0492$ & 0,1115 & $-0,4410$ & 0,6595 & $-04.80 \%$ \\
\hline
\end{tabular}

TABELA 2 - Parâmetros ajustados para a modelagem da média da precipitação no modelo GAMLSS com resposta Gama Generalizada para A2 - Porto Guarani.

\begin{tabular}{crrrrr}
\hline \multicolumn{2}{c}{ Parâmetro Estimativa } & $\begin{array}{c}\text { Erro } \\
\text { padrão }\end{array}$ & Estatistica $t$ & Valor-p & Efeito \\
\hline$\mu$ & 5,3687 & 0,0703 & 76,3253 & $<0,0001$ & - \\
$\beta$ & $-0,0102$ & 0,0022 & $-4,6665$ & $<0,0001$ & $-01,01 \%$ \\
$\alpha \mathrm{Fev}$ & $-0,1437$ & 0,1047 & $-1,3714$ & 0,1710 & $-13,39 \%$ \\
$\alpha \mathrm{Mar}$ & $-0,4043$ & 0,0914 & $-4,4222$ & $<0,0001$ & $-33,26 \%$ \\
$\alpha$ Abr & $-0,3509$ & 0,1172 & $-2,9932$ & 0,0029 & $-29,59 \%$ \\
$\alpha \mathrm{Mai}$ & $-0,0663$ & 0,1244 & $-0,5332$ & 0,5942 & $-06,41 \%$ \\
$\alpha$ Jun & $-0,2869$ & 0,1239 & $-2,3157$ & 0,0211 & $-24,94 \%$ \\
$\alpha$ Jul & $-0,3418$ & 0,1252 & $-2,7300$ & 0,0066 & $-28,95 \%$ \\
$\alpha$ Ago & $-0,7099$ & 0,1361 & $-5,2153$ & $<0,0001$ & $-50,83 \%$ \\
$\alpha$ Set & $-0,1307$ & 0,1185 & $-1,1031$ & 0,2706 & $-12,25 \%$ \\
$\alpha$ Out & 0,1195 & 0,1006 & 1,1884 & $0 ., 2354$ & $12,69 \%$ \\
$\alpha$ Nov & $-0,2236$ & 0,0982 & $-2,2776$ & 0,0233 & $-20,04 \%$ \\
$\alpha$ Dez & 0,0398 & 0,1045 & 0,3812 & 0,7033 & $04,06 \%$ \\
\hline
\end{tabular}

TABELA 3 - Parâmetros ajustados para a modelagem da média da precipitação no modelo GAMLSS com resposta Gama Generalizada para A3 - Balsa Santa Maria.

\begin{tabular}{crrrrrr}
\hline Parâmetro & Estimativa & $\begin{array}{c}\text { Erro } \\
\text { padrão }\end{array}$ & Estatisticat & Valor-p & Efeito \\
\hline$\mu$ & 5,3141 & 0,0694 & 76,5497 & $<0,0001$ & - \\
$\beta$ & $-0,0089$ & 0,0021 & $-4,3253$ & $<0,0001$ & $-00,89 \%$ \\
$\alpha$ Fev & $-0,1276$ & 0,1005 & $-1,2689$ & 0,2052 & $-11,98 \%$ \\
$\alpha$ Mar & $-0,4029$ & 0,0878 & $-4,5908$ & $<0,0001$ & $-33,16 \%$ \\
$\alpha$ Abr & $-0,3214$ & 0,1078 & $-2,9817$ & 0,0030 & $-27,49 \%$ \\
$\alpha$ Mai & $-0,0360$ & 0,1187 & $-0,3032$ & 0,7619 & $-03,54 \%$ \\
$\alpha$ Jun & $-0,3527$ & 0,1238 & $-2,8488$ & 0,0046 & $-29,72 \%$ \\
$\alpha$ Jul & $-0,5403$ & 0,1183 & $-4,5666$ & $<0,0001$ & $-41,74 \%$ \\
$\alpha$ Ago & $-0,6370$ & 0,1415 & $-4,5002$ & $<0,0001$ & $-47,11 \%$ \\
$\alpha$ Set & $-0,1593$ & 0,1143 & $-1,3936$ & 0,1642 & $-14,73 \%$ \\
$\alpha$ Out & 0,0796 & 0,0990 & 0,8043 & 0,4217 & $08,29 \%$ \\
$\alpha$ Nov & $-0,1560$ & 0,0938 & $-1,6618$ & 0,0973 & $-14,44 \%$ \\
$\alpha$ Dez & 0,0401 & 0,0989 & 0,4061 & 0,6849 & $04,09 \%$ \\
\hline
\end{tabular}

anteriormente destacam-se por apresentarem redução na precipitação pluviométrica média mensal da bacia hidrográfica do rio Piquiri.

As tabelas 4, 5 e 6 mostram os parâmetros ajustados para a modelagem da variância da precipitação no modelo GAMLSS com resposta Gama Generalizada para as três áreas de drenagem. Observa-se que a variabilidade da precipitação nos meses de maio a setembro é maior do que a variabilidade da precipitação do mês de janeiro.

CORREA \& GALVANI (2019) discutiram que neste período ocorrem os maiores e menores totais pluviométricos mensais para a área, destacando maio e setembro com anomalias positivas significativas de precipitação, enquanto o trimestre junho a agosto apresenta os principais desvios negativos de precipitação.

Observando a tabela 6 , que representa uma maior área de drenagem para a bacia hidrográfica do rio Piquiri, nota-se que para o parâmetro de dispersão da Precipitação, associado ao aumento na variância, não se verificou influência significativa proporcionada pelo IOS (valor- $p=78 \%$ ). Por outro lado, são significativos ao nível de $5 \%$ os aumentos de variabilidade identificados nos meses de maio a setembro.

A partir de maio observa-se um aumento de $40 \%$ na variabilidade da quantidade de chuvas com relação a variabilidade das chuvas nos meses de janeiro, mas este número chega a atingir $80 \% \mathrm{em}$ agosto, com uma diminuição dessa discrepância no mês de setembro, último mês com variação significativa e em que o aumento estimado foi de $33,08 \%$ (Tabela 6).

\section{CONSIDERAÇÕES FINAIS}

As análises mostraram que $31,4 \%$ dos meses da série histórica tiveram IOS negativo $\leq-7$ (indicando fase quente - El Niño), enquanto $21,1 \%$ dos meses obtiveram IOS positivo $\geq+7$ (indicando fase fria - La Niña), e 47,5\% dos meses foram considerados neutros com relação ao IOS.

As correlações mostraram que o IOS influencia a precipitação na bacia hidrográfica do rio Piquiri, com maior confiança, com um mês de defasagem, ou seja, os desvios positivos ou negativos no IOS levam em média um mês para repercutir na precipitação pluviométrica sobre a bacia hidrográfica do rio Piquiri.

O modelo GAMLSS mostrou-se satisfatório para a determinação da influência do IOS na precipitação. A análise dos resíduos apresentou-se 
TABELA 4 - Parâmetros ajustados para a modelagem da variância da precipitação no modelo GAMLSS com resposta Gama Generalizada para A1 - Guampará

\begin{tabular}{|c|c|c|c|c|c|}
\hline Parâmetro & Estimativa & $\begin{array}{c}\text { Erro } \\
\text { padrão }\end{array}$ & Estatística $t$ & Valor-p & Efeito \\
\hline$\tau$ & $-0,9996$ & 0,1274 & $-7,8453$ & $<0.0001$ & \\
\hline$\beta 2$ & 0,0001 & 0,0033 & 0,0222 & 0.9823 & $00,01 \%$ \\
\hline$\gamma \mathrm{Fev}$ & 13 & 1751 & & .1473 & $28,96 \%$ \\
\hline$\gamma$ Mar & $-0,0539$ & 0,1791 & $-0,3010$ & 0.7636 & $-05,25 \%$ \\
\hline & & 0,1771 & & 0.5226 & $12,01 \%$ \\
\hline$\gamma$ Mai & 4598 & 0,1721 & 6711 & 0.0080 & $58,38 \%$ \\
\hline$\gamma$ Jun & & 0,1740 & & 0.0522 & $40,38 \%$ \\
\hline$\gamma \mathrm{Jul}$ & & & & 0.0204 & $49,69 \%$ \\
\hline$\gamma$ Ago & & & & 0,0003 & $86,88 \%$ \\
\hline & & 0,1723 & 2,5792 & 0.0104 & $55,97 \%$ \\
\hline$\gamma$ Out & 0,2031 & 0,1758 & 1,1554 & 0.2488 & $22,52 \%$ \\
\hline & 0,0762 & 0,1775 & 0,4295 & 0.6678 & $07,92 \%$ \\
\hline$\gamma \mathrm{Dez}$ & 0,1798 & 0,1762 & 1,0202 & 0.3084 & $19,70 \%$ \\
\hline
\end{tabular}

TABELA 5 - Parâmetros ajustados para a modelagem da variância da precipitação no modelo GAMLSS com resposta Gama Generalizada para A2 - Porto Guarani

\begin{tabular}{crrrrr}
\hline Parâmetro Estimativa & $\begin{array}{c}\text { Erro } \\
\text { padrão }\end{array}$ & Estatistica t & Valor-p & Efeito \\
\hline$\tau$ & $-0,8897$ & 0,1113 & $-7,9929$ & $<0,0001$ & - \\
$\beta 2$ & 0,0021 & 0,0029 & 0,729 & 0,4664 & $00,21 \%$ \\
$\gamma$ Fev & 0,1131 & 0,1549 & 0,7303 & 0,4657 & $11,97 \%$ \\
$\gamma$ Mar & $-0,1666$ & 0,1578 & $-1,0557$ & 0,2917 & $-15,35 \%$ \\
$\gamma$ Abr & 0,3083 & 0,1525 & 2,0210 & 0,0439 & $36,11 \%$ \\
$\gamma$ Mai & 0,3984 & 0,1514 & 2,6319 & 0,0088 & $48,94 \%$ \\
$\gamma$ Jun & 0,3901 & 0,1513 & 2,5777 & 0,0103 & $47,71 \%$ \\
$\gamma$ Jul & 0,4074 & 0,1512 & 2,6939 & 0,0074 & $50,29 \%$ \\
$\gamma$ Ago & 0,5220 & 0,1496 & 3,4891 & 0,0005 & $68,54 \%$ \\
$\gamma$ Set & 0,3248 & 0,1523 & 2,1331 & 0,0335 & $38,38 \%$ \\
$\gamma$ Out & 0,0377 & 0,1557 & 0,2422 & 0,8088 & $03,84 \%$ \\
$\gamma$ Nov & $-0,0075$ & 0,1562 & $-0,0479$ & 0,9619 & $-00,75 \%$ \\
$\gamma$ Dez & 0,1097 & 0,1549 & 0,7083 & 0,4792 & $11,59 \%$ \\
\hline
\end{tabular}

TABELA 6 - Parâmetros ajustados para a modelagem da variância da precipitação no modelo GAMLSS com resposta Gama Generalizada para A3 - Balsa Santa Maria

\begin{tabular}{crrrrr}
\hline Parâmetro Estimativa & $\begin{array}{c}\text { Erro } \\
\text { padrão }\end{array}$ & Estatística $t$ & Valor-p & Efeito \\
\hline$\tau$ & $-0,9049$ & 0,1094 & $-8,2735$ & $<0,0001$ & - \\
$\beta 2$ & 0,0008 & 0,0029 & 0,2691 & 0,7880 & $00.08 \%$ \\
$\gamma$ Fev & 0,0620 & 0,1527 & 0,4057 & 0,6852 & $06.40 \%$ \\
$\gamma$ Mar & $-0,2387$ & 0,1560 & $-1,5298$ & 0,1268 & $-21,23 \%$ \\
$\gamma$ Abr & 0,1895 & 0,1511 & 1,2540 & 0,2105 & $20,86 \%$ \\
$\gamma$ Mai & 0,3446 & 0,1491 & 2,3114 & 0,0213 & $41,14 \%$ \\
$\gamma$ Jun & 0,4067 & 0,1482 & 2,7446 & 0,0063 & $50,19 \%$ \\
$\gamma$ Jul & 0,3395 & 0,1491 & 2,2765 & 0,0233 & $40,42 \%$ \\
$\gamma$ Ago & 0,5912 & 0,1459 & 4,0510 & 0,0001 & $80,62 \%$ \\
$\gamma$ Set & 0,2858 & 0,1498 & 1,9073 & 0,0572 & $33,08 \%$ \\
$\gamma$ Out & 0,0331 & 0,1530 & 0,2160 & 0,8291 & $03,37 \%$ \\
$\gamma$ Nov & $-0,0766$ & 0,1543 & $-0,4961$ & 0,6201 & $-07,37 \%$ \\
$\gamma$ Dez & 0,0294 & 0,1531 & 0,1923 & 0,8476 & $02,98 \%$ \\
\hline \multicolumn{5}{c}{} &
\end{tabular}

dentro do esperado, e conclui-se que conforme aumenta uma unidade no IOS (tende a ser positivo - indicador da fase fria), a precipitação média diminui $1 \%$ na bacia hidrográfica.

A variação do efeito do IOS na redução da precipitação entre as três áreas de drenagem demonstra uma variabilidade espacial e, quando se considera outras características do clima, percebese que a área da bacia hidrográfica por estar em uma faixa de transição entre o clima tropical e subtropical responde aos efeitos do fenômeno ENOS como já identificado pela literatura.

\section{AGRADECIMENTOS}

Aos relatores da Revista do Instituto Geológico pelas sugestões apresentadas que enriqueceram o artigo.

\section{REFERÊNCIAS BIBLIOGRÁFICAS}

AMBRIZZI, T. 2003. El nino/oscilações sul e teleconexões atmosféricas no hemisfério austral. Universidade de São Paulo, São Paulo, Tese de Livre-Docência.

\section{BOM-BUREAU OF METEOROLOGY. Southern} Oscillation Index. Disponível em http://www. bom.gov.au/climate/enso/soi/about-soi.html. Acessado em 09 de dez. 2020.

COELHO, C.A.S.; UVO, C.B.; AMBRIZZI, T. 2002. Exploring the impacts of the Tropical Pacific SST on the precipitation patterns over South America during ENSO periods. Theoretical and Applied Climatology, 71: 185-197. https://doi.org/10.1007/ s007040200004

CORREA, M.G.G. 2013. Distribuição espacial e variabilidade da precipitação pluviométrica na bacia do rio Piquiri-PR. Faculdade de Filosofia, Letras e Ciências Humanas, Universidade de São Paulo, São Paulo, Dissertação de Mestrado, 104 p. http://dx.doi. org/10.11606/D.8.2013.tde-28082013122143

CORREA, M.G.G.; GALVANI, E. 2019. Correlação e variabilidade da precipitação e a vazão na bacia hidrográfica do rio Piquiri - PR. Geo UERJ, 34: e, 40945. https://doi. org/10.12957/geouerj.2019.40945 
DANTAS，L.G.; SANTOS, C.A.C.; OLINDA, R.A.; BRITO, J.I.B.; SANTOS, C.A.G.; MARTINS, E.S.P.R.; DE OLIVEIRA, G.; BRUNSELL, N.A. 2020. Rainfall Prediction in the State of Paraíba, Northeastern Brazil Using Generalized Additive Models. Water, 12: 2478. https://doi. org/10.3390/w12092478

DEBELE, S.E.; BOGDANOWICZ, E.; STRUPCZEWSKI, W.G. 2017. Around and about an application of the GAMLSS package to non-stationary flood frequency analysis. Acta Geophysica, 65: 885-892. https://doi.org/10.1007/s11600-017-0072-3

FERREIRA, A.G. 2006. Meteorologia prática. Oficina de Textos, São Paulo, 192 p.

GRIMM, A.M.; BARROS, V.R.; DOYLE, M.E. 2000. Climate variability in Southern South America associated with El Niño and La Niña events. Journal of Climate, 13: 35-58. https:// doi.org/10.1175/1520-0442(2000)013\%3C0 035:CVISSA\%3E2.0.CO;2

IAPAR - INSTITUTO AGRONÔMICO DO PARANÁ. 1978. Cartas climáticas do Estado do Paraná. IAPAR, Londrina.

IBGE - INSTITUTO BRASILEIRO DE GEOGRAFIA E ESTATÍSTICA. 2006. Censo agropecuário 2006. IBGE, Rio de Janeiro.

IBGE - INSTITUTO BRASILEIRO DE GEOGRAFIA E ESTATÍSTICA. 2012. Censo Brasileiro de 2010. IBGE, Rio de Janeiro.

JEHANZAIB, M.; SHAH, S.A.; YOO, J.; KIM, T.W. 2020. Investigating the impacts of climate change and human activities on hydrological drought using non-stationary approaches. Journal of Hydrology, 588: 125052. https://doi.org/10.1016/j. jhydrol.2020.125052

KÖPPEN, W. 1948. Climatologia: com un estúdio de los climas de la tierra. Ed. Fondo de Cultura Econômica, México, 478 p.

LANNA, A.E. 2001. Elementos de estatística e probabilidades. In: C.E.M Tucci (ed.) Hidrologia: Ciência e aplicação. Porto Alegre, Ed. da Universidade - UFRGS, ABRH, p. 79-176.
LIMBERGER, L.; SILVA, M.E.S. 2016. Precipitação na bacia amazônica e sua associação à variabilidade da temperatura da superfície dos oceanos Pacífico e Atlântico: uma revisão. GEOUSP: Espaço e Tempo, 20(3): 657-675. https://doi.org/10.11606/ issn.2179-0892.geousp.2016.105393

MILHORANÇA, I.A. 2014. Modelos paramétricos para séries temporais de contagem. Instituto de Matemática e Estatística, Universidade de São Paulo, São Paulo, Dissertação de Mestrado, $71 \mathrm{p}$.

MOLION, L.C.B.; BERNARDO, S.O. 2000. Dinâmica das chuvas no Nordeste brasileiro. In: SBMET, CONGRESSO BRASILEIRO DE METEOTROLOGIA, 11, Rio de Janeiro, Anais.

NERY, J.T. 2005. Dinâmica climática da região sul do Brasil. Revista Brasileira de Climatologia, 1(1): 61-75. http://dx.doi.org/10.5380/ abclima.v1i1.25233

PAIVA, C.S.M.; FREIRE, D.M.C.; CECATTI, J.G. 2008. Modelos aditivos generalizados de posição, escala e forma (GAMLSS) na modelagem de curvas de referência. Revista Brasileira de Ciências da Saúde, 12(3): 289310. https://periodicos.ufpb.br/ojs/index. $\mathrm{php} / \mathrm{rbcs} /$ article/view/4465

REBOITA, M.S.; SANTOS, I.A. 2014. Influência de alguns padrões de teleconexões na precipitação do Norte e Nordeste do Brasil. Revista Brasileira de Climatologia, 15: 28-48. http:// dx.doi.org/10.5380/abclima.v15i0.37686

RODRIGUES, C.; ADAMI, S. 2005. Técnicas fundamentais para o estudo de bacias hidrográficas. In: L.A.B. Venturi (org.) Praticando Geografia: técnicas de campo e laboratório em geografia e análise ambiental. São Paulo, Oficina de Textos, p. 147-166.

SCHNEIDER, C.; GIES, D. 2004. Effects of El Niño-Southern oscillation on Southernmost South America precipitation at $53^{\circ} \mathrm{S}$ revealed from Ncep-Ncar reanalyses and weather station data. International Journal of Climatology, 24: 1057-1076. http://dx.doi. org/10.1002/joc. 1057 
SEMA - SECRETARIA DE ESTADO DO MEIO AMBIENTE E RECURSOS HÍDRICOS. 2010. Bacias hidrográficas do Paraná: Série histórica. Governo do Estado do Paraná.

SILVA, C.B. 2012. Variabilidade climática nos oceanos e a vazão fluvial no Planalto Brasileiro. Faculdade de Filosofia, Letras e Ciências Humanas, Universidade de São Paulo, São Paulo, Dissertação de Mestrado, 201 p. http://dx.doi.org/10.11606/D.8.2012. tde-27022013-092155
SILVA, M.E.S.; SILVA, C.B. 2012. Variabilidade climática - Processos físicos e dinâmicos nos oceanos e atmosfera. Revista do Departamento de Geografia, Volume Especial 30 anos: 372-406. https://doi. org/10.7154/RDG.2012.0112.0016

STASINOPOULOS, D. M.; RIGBY, R. A. 2007. Generalized additive models for location scale and shape (GAMLSS). R. Journal of Statistical Software, 23(7): 1-46. http:// dx.doi.org/10.18637/jss.v023.i07

\section{Endereço dos autores:}

Márcio Greyck Guimarães Correa - Diretoria de Ensino, Região Centro Oeste, Secretaria da Educação do Estado de São Paulo, Av. Rio Branco, 1260, Campos Elíseos, CEP 01206-001, São Paulo, SP, Brasil. E-mail:mgreyckcorrea@gmail.com

Emerson Galvani - Departamento de Geografia, Faculdade de Filosofia, Letras e Ciências Humanas, Universidade de São Paulo, Av. Lineu Prestes, 338, CEP 05508-000, Cidade Universitária, São Paulo, SP, Brasil.E-mail: egalvani@usp.br

Artigo submetido em 10 de dezembro de 2020, aceito em 29 de dezembro de 2020. 